ÉGYPTE

monde arabe

\section{Égypte/Monde arabe}

5-6| 2009

Pratiques du Patrimoine en Égypte et au Soudan

\title{
Processus et enjeux de la patrimonialisation de la musique copte
}

Processes and matters of making Coptic music a heritage

\section{Séverine Gabry}

\section{(2) OpenEdition}

\section{Journals}

Édition électronique

URL : https://journals.openedition.org/ema/2896

DOI : 10.4000/ema.2896

ISSN : 2090-7273

Éditeur

CEDEJ - Centre d'études et de documentation économiques juridiques et sociales

Édition imprimée

Date de publication : 22 décembre 2009

Pagination : 133-158

ISBN : 2-905838-43-4

ISSN : 1110-5097

Référence électronique

Séverine Gabry, "Processus et enjeux de la patrimonialisation de la musique copte », Égypte/Monde arabe [En ligne], 5-6 | 2009, mis en ligne le 31 décembre 2010, consulté le 07 juillet 2022. URL : http:// journals.openedition.org/ema/2896 ; DOI : https://doi.org/10.4000/ema.2896 


\section{RÉSUMÉ / ABSTRACT}

\section{PROCESSUS ET ENJEUX DE LA PATRIMONIALISATION DE LA MUSIQUE COPTE}

L'étude des minorités chrétiennes en terre d'Islam est fondamentale pour la compréhension des différents processus à l'œuvre dans ces sociétés secouées par de fortes querelles identitaires. Parmi ces minorités, la communauté copte d'Égypte (entre 6 et $10 \%$ de la population) offre un champ d'étude privilégié. Cet article analyse dans une perspective sociologique le cas de la musique copte, essentiellement liturgique, ainsi que le discours identitaire qui $s^{\prime} y$ attache. Cette démonstration des pratiques musicales s'articule autour de la notion de tradition, dont la récurrence dans le discours des acteurs nous pousse à examiner le sens, ainsi que les moyens de diffusion mis en place pour faire de cette musique un patrimoine à part entière.

\section{PROCESSES AND MATTERS OF MAKING COPTIC MUSIC A HERITAGE}

The study of Christian minorities in Islamic societies is fundamental to understand diverse processes at work in these societies characterised by strong identity disputes. Among these minorities, the Coptic community in Egypt (between 6 and $10 \%$ of the population) provides a unique field of study. This article explores in a sociological perspective, the case of Coptic music, mostly liturgical, and the related discourse of identity. The display of musical practices is built on the concept of tradition, whose recurrence in the actors' speech leads us to consider the meaning and the means of dissemination orchestrated to give this music a full heritage status. 

PROCESSUS ET ENJEUX

\section{DE LA PATRIMONIALISATION \\ DE LA MUSIQUE COPTE}

Coptic Music is one of the most precious Original Egyptian arts. It is oldest Musical Heritage ever known world-wide. It is a folklore having very old Egyptians roots. That's why, it is a duty of all Egyptians to preserve it.

Ragheb Moftah, Coptic Music (1995).

D

epuis le XIX ${ }^{e}$ siècle, les Coptes d'Égypte réalisent de nombreux travaux sur leur histoire et leurs pratiques, particulièrement dans l'optique d'asseoir la légitimité de la communauté et de se doter d'une certaine visibilité. C'est en effet dès 1811 que Muhammad 'Alî (1769-1849)¹ octroie de nouveaux droits aux Coptes, jusqu'alors opprimés par le pouvoir mamelouk. L'Église Copte entreprend dès lors un long processus de renouvellement et, en parallèle, de constitution de son patrimoine culturel. Ce renouveau est lancé par le pape Cyrille IV (Pape de l'Église copte d'Alexandrie de 1853 à 1861), qui pense que I'éducation est primordiale à la renaissance de son peuple. ${ }^{2}$ Les pratiques liturgiques occupant une place prépondérante dans la vie des Coptes, elles sont devenues dès cette époque l'un des vecteurs de la mise en valeur de ce que cette communauté appelle la «culture copte ». Quant à la musique liturgique, dont le rôle est central dans l'office religieux, elle est à présent la clé de voûte non seulement de la foi copte mais aussi de sa représentation.

Pour comprendre comment les chrétiens d'Égypte mettent aujourd'hui en valeur leur musique, il est nécessaire de travailler à leurs côtés. II a fallu pour

1. Général albanais très ambitieux qui prit le pouvoir en Égypte dans le sillage de Bonaparte et se maintint sur le trône pendant 44 ans, 'Afaf Lutfî al-Sayyid Marsot, 1984, Egypt in the reign of Muhammad 'Ali, Cambridge, Cambridge Middle East Library.

2. À ce sujet, voir le paragraphe concernant la musique Copte dans la thèse de Bernard Moussali, Les enregistrements du Congrès de Musique arabe du Caire, recueilli et édité par Jean Lambert. À paraître. 
cela réaliser une première mission de terrain au Caire (menée durant le mois de février 2007), qui m'a permis de me familiariser avec la musique copte telle que pratiquée et transmise aujourd'hui dans la capitale égyptienne. Ce fut également l'occasion de confronter la musique copte à sa pratique actuelle et aux idées communément admises sur son ancienneté et son origine pharaonique, et surtout à son rôle dans le processus de construction identitaire de cette communauté depuis un siècle. ${ }^{3}$

Il est remarquable de constater, à travers cette étude ethnographique, que les travaux sur l'authenticité de la musique liturgique copte ${ }^{4}$ ont eu pour conséquence de créer une sorte de tradition figée à un moment dans le temps. Les auteurs de ces écrits ne sont sans doute pas les seuls à avoir contribué à développer cette idée, mais l'authenticité présumée de la musique liturgique renvoie nettement à la volonté qu'ont les Coptes d'être les détenteurs d'une tradition millénaire. Cet aspect apparaît dans le discours de personnalités influentes et connues des Chrétiens d'Égypte - comme le chef de chœur George Kyrillos - et dans certains ouvrages. ${ }^{5}$

Pendant mes premières investigations menées en février 2007, j’ai pu cerner deux aspects essentiels de la pratique musicale copte au Caire : I'existence de processus de fabrication du patrimoine musical copte, et les enjeux de la sauvegarde de ce même patrimoine. En effet, la patrimonialisation de cette musique, opérée par le biais de sa transmission, de son archivage, de sa commercialisation, etc. renvoie essentiellement au besoin qu'ont certains Coptes de se mettre en valeur en tant que communauté à part entière. Des enjeux identitaires stratégiques et la présence d'un consensus politique et social au sein de ce qui était parfois appelé par certains la « nation copte ${ }^{6}$ ressortent

3. Ces idées sont notamment celles de Hans Hickmann, Ilona Borsai et René Ménard. Ils ont tous trois étudié la musique copte en lui conférant une descendance pharaonique indéniable, sous le prétexte discutable que les Coptes seraient les vrais égyptiens, purs héritiers des traditions de l'Égypte ancienne. Ce type de discours contribue amplement à développer un sentiment nationaliste très fort chez les Coptes et semble étroitement lié à cette volonté de mettre en valeur la «culture Copte».

4. Ces travaux sont une fois encore ceux de H. Hickmann, I. Borsai et R. Ménard, cités en bibliographie. Ils concourent tous à l'idée que la musique copte descend directement de la musique pharaonique.

5. Le discours général copte demeure nettement apologétique envers sa culture et ses antécédents, comme nous avons pu le constater dans de diverses revues coptes récentes (les Cahiers Coptes, Le Monde Copte,...), la Coptic Encyclopedia et I'ouvrage de George Kyrillos, Coptic hymnody, its Spirituality and Tunes, à consulter sur le site : [http://www.davidensemble.com/english/book.htm].

6. C'est ainsi que certains groupes de jeunes étudiants chrétiens s'étaient définis au début des années 1950, al-Umma al-qibtiyya, revendiquant pour leur religion le statut d'un état, Voile, 2004. 
ainsi de cette sauvegarde musicale. La musique devient en ce sens un bien unique, représentatif de la singularité des Coptes par rapport aux Égyptiens Musulmans.

$\mathrm{C}^{\prime}$ est depuis le renouveau du XIXe siècle que des investissements à la fois intellectuels et financiers se développent pour conserver, préserver, mettre en valeur et promouvoir le patrimoine musical des Coptes. Ce sont la transmission par la création d'écoles de musique et d'instituts, le développement d'enregistrements et de moyens de diffusion de la musique et la réalisation de transcriptions. Pour les chrétiens qui chantent ou jouent les hymnes, ces démarches sont liées à une mise au jour des constituants de leur culture dans le cadre d'un rapport de domination musulmane qui s'exacerbe en Égypte. ${ }^{7}$ Elles sont également facilitées par un regard occidental favorable aux Coptes, notamment par le caractère mythique de l'Égypte qui, depuis l'Expédition de Bonaparte (1798-1801), n'a cessé de susciter la plus grande fascination auprès des étrangers. On relèvera à titre d'exemple les concerts de musique copte réalisés à l'étranger notamment par l'Ensemble David, chorale dirigée par George Kyrillos. La plupart des manifestations musicales auxquelles le chœur a participé étaient liées au désir de faire revivre la musique des anciens Égyptiens par le biais des Coptes. Citons à ce propos I'Institut du Monde Arabe : I'Ensemble David a été invité à interpréter certains de ses chants dans le cadre des représentations liées aux musiques traditionnelles soufies. L'intitulé de ces concerts était « 2000 ans de chants et de musique spirituelle en Égypte». Lors du «Festival de l'Orient » à Stockholm en 1997, G. Kyrillos a été convié afin de présenter les hymnes chrétiens et d'expliquer leurs caractéristiques par rapport à la musique pharaonique. II a également interprété I'hymne "Golgotha », dit d'ascendance pharaonique, accompagné par l'orchestre philharmonique de Sofia et le chœur de Venise à l'Opéra de Marseille et à la Basilique Saint-Denis à Paris en 1998. Ces manifestations en Europe trahissent le goût occidental pour des cultures considérées porteuses d'une histoire prestigieuse et dites inchangées depuis des millénaires. Une telle vision conforte la volonté des Coptes de promouvoir leur patrimoine musical et d'éviter ainsi sa dégradation présumée.

La musique copte désigne initialement la musique pratiquée lors du culte. Elle se caractérise par des chants a capella, monodiques et interprétés par des hommes, à l'exception des répons chantés par toute l'assemblée. Le seul accompagnement instrumental de l'office est rythmique et exécuté, à I'heure actuelle, par des cymbales et un triangle. En général, la représentation actuelle de la musique copte reste ancrée dans son rapport avec la liturgie. Depuis que cette église existe, son culte se chante. Diacres, prêtres, chantres et fidèles

7. À ce sujet, voir par exemple : Christian Cannuyer, Les Coptes, Belgique, Brepols, 1996, collection «Fils d'Abraham ». 
participent à ce grand théâtre spirituel. La conservation de leur musique souligne cet investissement des Coptes dans leur vie religieuse.

Plus que la crainte d'un oubli, c'est celle d'une dégradation d'une musique « authentique » qui a engendré le développement de processus de patrimonialisation. Pour les Coptes interrogés, le temps menaçait de dégrader et de transformer leur patrimoine musical. C'est ainsi qu'ils justifient leur besoin de conserver concrètement leurs chants, pour eux seule trace tangible de leur glorieux passé à même de se maintenir dans le présent. Les premières ébauches de conservation commencent avec la transcription des chants et l'éducation des jeunes Coptes. Puis, dès l'apparition des premiers phonographes, conserver cette musique et pouvoir la réécouter devient possible. Rappelons qu'à la fin du XIXe siècle, les évènements politiques en Égypte agissent favorablement sur les aspirations éducatives de la communauté copte. L'action du patriarche Cyrille IV permet la création d'écoles, d'une imprimerie, etc. Les droits que les Coptes obtiennent progressivement sous Muhammad ${ }^{\mathrm{c} A}$ lî (accès au service militaire, abolition de la gizyah, impôt spécifiquement copte) les élèvent au rang de citoyens à part entière. Ils sont reconnus égaux aux Musulmans devant la loi, ce qui n'était pas le cas avant.

En Égypte, les trois processus majeurs qui découlent de ce renouveau prôné dès le XIX ${ }^{\text {e }}$ siècle sont la transcription d'une musique de tradition orale, la transmission aux générations futures dans le cadre d'un apprentissage institutionnalisé et le développement d'enregistrements de musiques, qu'elles soient liturgiques ou non. Ces moyens permettent également de divulguer cette musique, en plus de la conserver.

\section{LA TRANSCRIPTION MUSICALE : FONCTION ET CONCEPTION}

Dès ses débuts, la transcription participe d'une forte volonté de sauvegarde de la musique et du souci de lutter contre son éventuelle dégradation. ${ }^{8}$ L'enjeu $^{\prime}$ de la notation est donc de préserver ce patrimoine sonore sans qu'il subisse la moindre modification, mais aussi de l'étudier et de l'analyser. Pour les Coptes, écrire les chants de la liturgie n'a toutefois jamais été en soi un objectif, car leur transmission s'est toujours effectuée par le biais de l'oralité ; noter n'est donc devenu un outil d'apprentissage que récemment, le phénomène n'apparaissant qu'à la fin du XIXe siècle parallèlement au renouveau prôné par Cyrille IV. Chanteurs et religieux sont dès lors désireux de laisser une trace pérenne de leur musique liturgique.

8. Les premiers essais de transcription ont été publiés au début du XIXe siècle : Guillaume André Villoteau, 1826, Description de l'Égypte, Paris, Imprimerie Impériale, tome XIV. L'objectif de Villoteau n'était pas, toutefois, de pallier la dégradation de cette musique pour laquelle il n'avait pas grande considération. 
Il est complexe de transcrire une musique qui participe autant de I'oralité, comme toutes les musiques dites «traditionnelles » qui laissent une large part à la spontanéité improvisatrice de l'exécutant. Des divergences entre les différents transcripteurs sont ainsi apparues sur le choix du type de notation. Certains optent, encore aujourd'hui, pour une écriture épurée et d'autres, pour une écriture enrichie de tous les ornements exécutés par le chantre. Si tous sont unanimes sur le caractère indispensable d'une telle démarche, les avis divergent quant à la manière de noter cette musique mélismatique ${ }^{9}$ et suscitent de nombreux débats ${ }^{10}$ sur la qualité d'une transcription par rapport à une autre. Dans le cadre de cette vaste entreprise de notation et de sauvegarde, tous les chants sont systématiquement enregistrés depuis une cinquantaine d'années. Ce travail auquel Ragheb Moftah (1898-2001) - professeur responsable de la section « musique » à I'Institut d'Études Coptes de 1954 à 2001 -, a donné une très forte impulsion tout au long du XXe siècle, est aujourd'hui poursuivi par de nouveaux acteurs, comme Michael Ghattas, responsable actuel de la section « musique » et George Kyrillos, chef du chœur l'Ensemble David, convaincus du bien-fondé de la démarche de leur prédécesseur.

Les Occidentaux ont abordé les questions de transcription musicale avant les Coptes. Cela ne signifie pas que les chrétiens d'Égypte n'ont jamais esquissé la moindre forme de notation par le passé. II existe en effet des manuscrits encore inexploités à étudier, qui présentent des signes au-dessus des mots visiblement sans rapport avec la langue copte. Ainsi, s'il est vrai que les Coptes n'ont développé la notation de leurs chants sur portées que récemment, notamment avec Ragheb Moftah et George Kyrillos, nous ne savons pas à l'heure actuelle si une quelconque notation ekphonétique n'a pas existé par le passé. «Ekphonétique » vient du grec ekphonesis, qui signifie «lecture à haute voix, solennelle ". II s'agit d'un système de notation apparu vers le VIII siècle, qui concerne davantage la prosodie que la musique et résulte de la lecture déclamée des textes scripturaux. Les signes peuvent indiquer la hauteur sur laquelle on lira un mot entier ou même plusieurs mots, mais ne précisent ni un intervalle ni une durée. ${ }^{11}$ René Ménard a essayé d'identifier les plus anciennes

9. Opposé à "syllabique », chanter de façon «mélismatique » consiste à réaliser de nombreux ornements sur une syllabe. Très utilisé dans le cadre de la liturgie, les chants mélismatiques seraient un moyen d'accéder au divin sans avoir recours à I'imperfection des mots.

10. Les questions suscitées par des divergences de point de vue liées au type de transcription sont notamment soulevées dans le livre de George Kyrillos, disponible sur le site internet de l'Ensemble David: George Kyrillos, Coptic hymnody, its Spirituality and Tunes : [http://www.davidensemble.com/english/book.htm].

11. Philippe Vigreux, 1992, Musique arabe - le Congrès du Caire de 1932, Le Caire, CEDEJ, p. 39. 
traces de la notation musicale copte, en prenant appui sur cette idée d'écriture ekphonétique. Les résultats sont toutefois assez succincts. II affirme qu'aucun traité de musique copte n'existe et que « notre documentation en manuscrits musicaux coptes est [...] extrêmement pauvre et [...] parfaitement inutilisable jusqu'à ce jour ». ${ }^{12}$ II signale certains de ces manuscrits dans son article "La musique Copte, problème insoluble? ». L'article sur la musique Copte, dans I'encyclopédie de Aziz S. Atiya (p. 1731-1732), fait également mention de deux manuscrits retrouvés, semblant présenter une notation ekphonétique. Le premier date de trois siècles avant la christianisation de l'Égypte et montre la plus ancienne trace présumée de notation musicale (Zenon, Musée du Caire, $\left.\mathrm{n}^{\circ} 59533\right)$; le second est le fragment $\mathrm{d}^{\prime}$ un hymne qui date du III $\mathrm{I}^{\mathrm{e}}$ siècle après J.-C., tiré du papyrus d’Oxyrhynchos 1786, découvert par Grenfell et Hunt. Ce dernier exemple pourrait être la plus ancienne trace d'une notation musicale chrétienne. Les premiers essais de transcription sur portées de ces musiques datent de l'Expédition d'Égypte (1798-1801), publiées par Guillaume André Villoteau (1759-1839) en 1826. ${ }^{13}$ Puis ce sont les Pères Jules Blin et Louis Badet, qui, respectivement en 1888 et 1899, se sont attelés à cette tâche. ${ }^{14}$ Depuis, de nombreux essais de transcriptions ont vu le jour, sous différentes formes, soulevant des interrogations au sein et en dehors de la communauté copte.

Les principales démarches égyptiennes de transcription ont été menées par Ragheb Moftah. Pour lui, noter la musique avait vocation de pallier sa dégradation, présumée imminente. II a ainsi fait appel à des chantres et à des musiciens musicologues pour l'aider dans son entreprise ${ }^{15}$ et a réalisé, dès lors, les premiers enregistrements avec les chantres qu'il avait choisis. Le premier musicologue qui a aidé R. Moftah était le professeur Ernest Newlandsmith, de la Royal Academy of Music, venu en Égypte sur son invitation en 1927. II fût alors chargé de transcrire en notation occidentale les chants de la liturgie, entreprise qu'il a poursuivie jusqu'en 1936. Seize volumes de transcription de E. Newlandsmith, inédits, sont conservés à la Library of Congress de Washington ${ }^{16}$ et une copie existe à I'Institut d'Études Coptes au Caire.

12. René Ménard, 1952, "La musique Copte, problème insoluble? ", Les Cahiers Coptes, tome I, p.35.

13. Guillaume André Villoteau, 1826, Description de l'Égypte, Paris, Impr. Impériale, tome XIV, chapitre V.

14. Cf. Infra, notes 29 et 33.

15. "Dr Moftah's search for cantors who knew the authentic melodies of the church began in 1926 ». Préface de A transcription of the Complete Liturgy of St Basile, Ragheb Moftah, avec la collaboration de Margit Tóth et Martha Roy, Le Caire, 1998. 16. L'initiative de transférer cette documentation est de R. Moftah. C'est par l'intermédiaire de Martha Roy et Marian Robertson que ces chants ont été envoyés aux États-Unis, et grâce aussi à l'aide de la nièce de R. Moftah, Laurence Moftah, professeur à I'Université américaine du Caire. De nombreux enregistrements s'y trouvent également. (Référence No.: ML31.M58). 

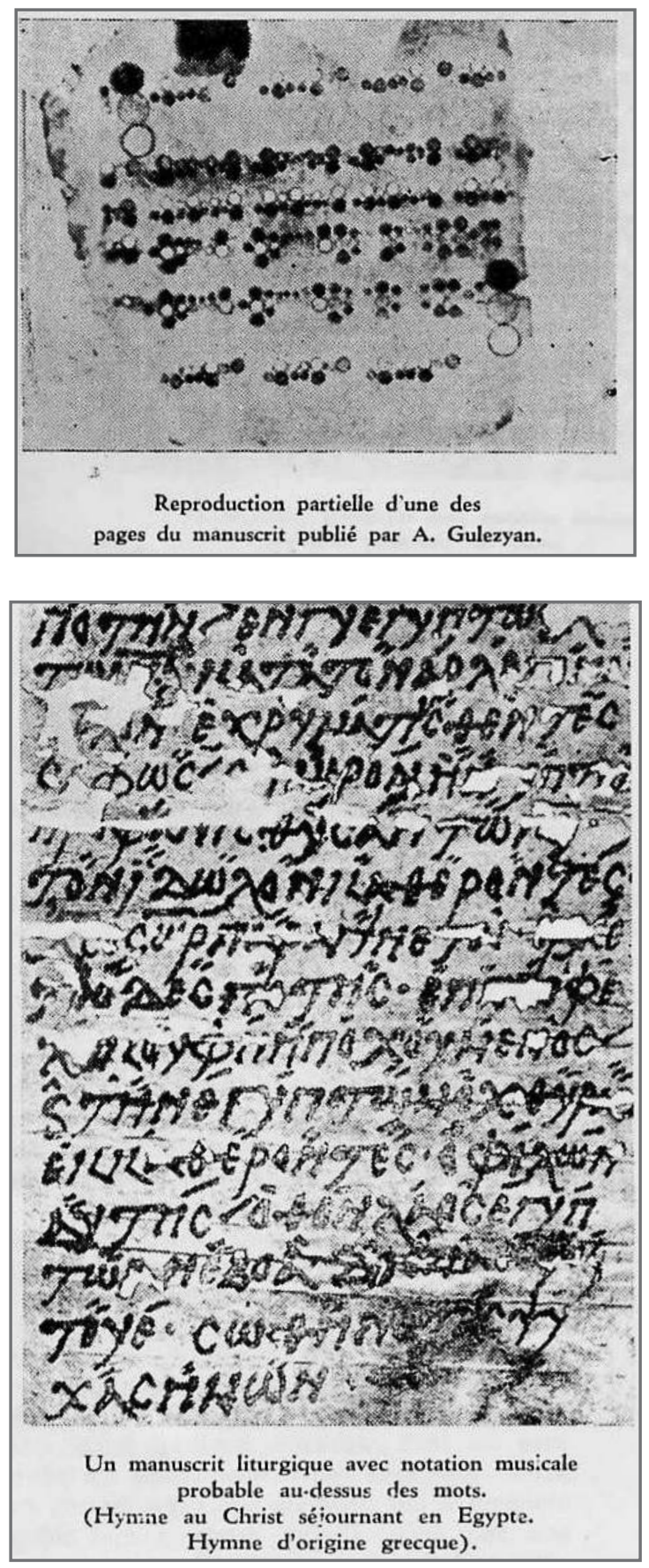

1. manuscrits cités par René Ménard en 1952 dans «La musique Copte, problème insoluble? », Les Cahiers Coptes, Le Caire, Institut d'Études Coptes, tome I. 
L'une des requêtes adressées par R. Moftah à E. Newlandsmith était de choisir parmi plusieurs chantres celui qui lui semblait être le réel dépositaire de la tradition musicale Copte. Suite aux différentes écoutes, il s'est avéré que cette personne était Mikhail Girgis el-Batanony, pour sa connaissance de chants que d'autres ignoraient et aussi pour son aptitude à réaliser plusieurs fois de suite la même mélodie, ce dont les autres n'étaient pas tous capables. Ce jugement montre une volonté de conserver l'héritage transmis par un exécutant considéré comme garant des traditions. Toutefois, cette idée a ses limites : une mélodie plusieurs fois répétée de manière identique n'implique pas l'existence d'une musique "authentique». Si elle n'est pas rigoureusement la même d'une exécution à l'autre, cela peut venir d'une liberté improvisatrice. Quant aux chants méconnus par certains chantres, y voir des mélodies traditionnelles perdues est une préconception, car il peut tout aussi bien s'agir de mélodies inventées. Trouver un « détenteur de la tradition » est ainsi une tentative complexe, qui implique d'autres problèmes : de quelle tradition s'agit-il et par rapport à quelle époque une exécution est-elle considérée comme traditionnelle ou non ? Dans le cas de la musique copte, la tradition a été admise comme invariable depuis 2000 ans, ce qui rend toute tentative de réponse difficile.

La liturgie de saint Basile ${ }^{17}$ en particulier est présentée dans l'un des volumes des transcriptions de E. Newlandsmith précités. Cette messe a fait I'objet d'une autre transcription quelques dizaines d'années plus tard, toujours sous l'impulsion de R. Moftah. Le chant copte regorge de mélismes et d'ornementations. Or, les notations du professeur anglais étaient épurées, ces derniers étant pour lui des "débris de musique arabe ". ${ }^{18}$ Au regard des objectifs de R. Moftah, ce travail semblait insuffisant en vue d'une bonne conservation du chant copte. C'est ainsi qu'il a travaillé, par la suite, avec Margît Tóth, chef de la section musicale du Musée d'ethnographie de Budapest, et Martha Roy, professeur à la faculté d'Éducation musicale d'Helwan, au Caire, afin de mener à bien l'archivage de la messe de saint Basile. M. Tóth a débuté son travail de transcription à partir des nombreux enregistrements de R. Moftah en 1968. Pour se faire, elle a adopté la technique de B. Bartók, qui consistait en une notation précise et détaillée du moindre élément musical perçu grâce, notamment, aux outils perfectionnés d'enregistrement dont elle disposait, qui

17. La liturgie de saint Basile - ascète, moine et théologien du IVe siècle - est la plus commune du rite copte. Elle aurait intégralement survécu grâce à la transmission orale pendant des siècles. On en possède à présent une transcription complète (chants et hymnes), éditée avec la collaboration de l'Université américaine du Caire (Moftah, 1998).

18. Ernest Newlandsmith, 12 mai 1931, The Ancient Music of the Coptic Church, conférence présentée à la University Church, Oxford. 


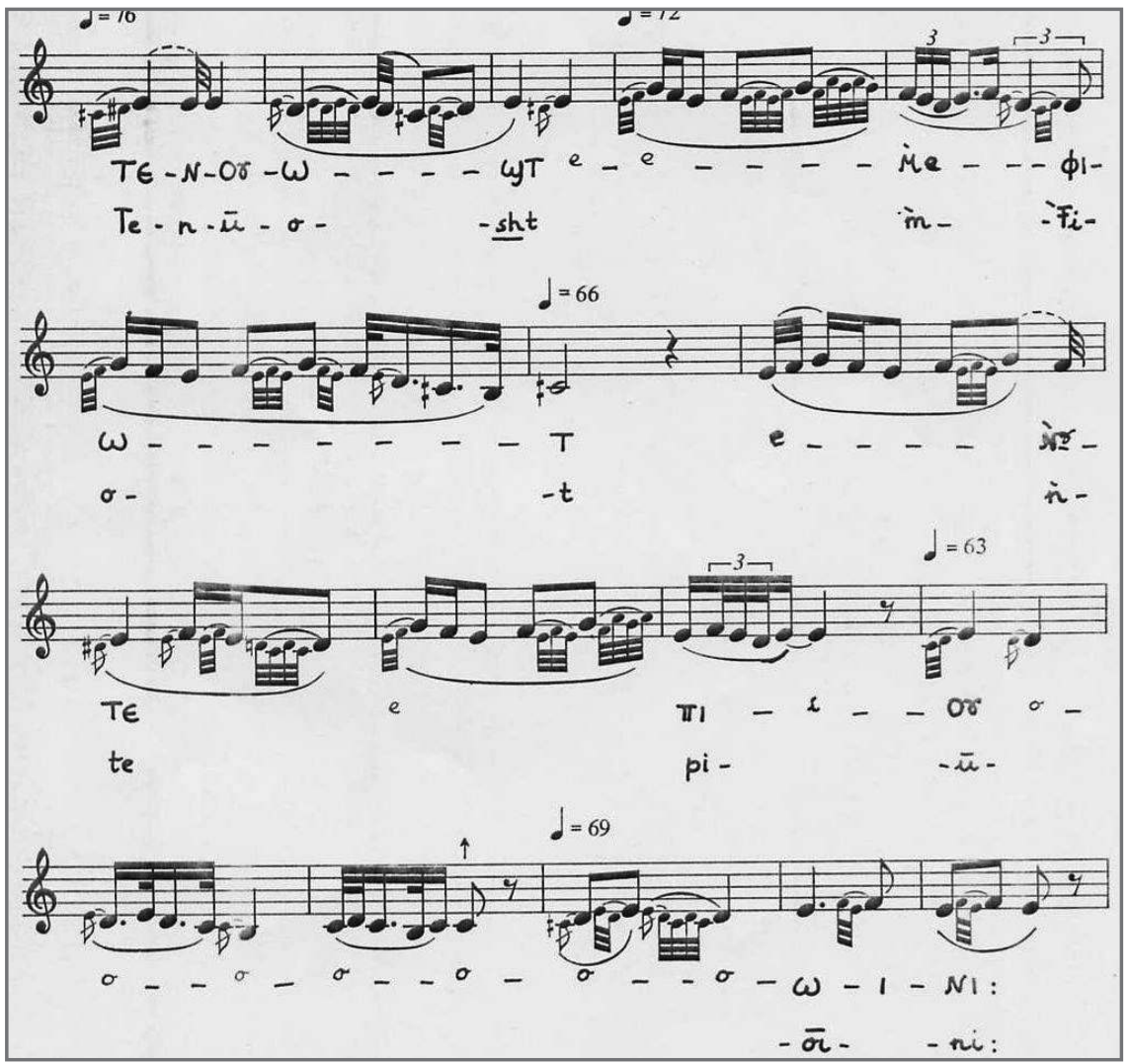

2. "Ten ouosht», fragment, liturgie de saint Basile, par Margît Tóth. A transcription of the Complete Liturgy of St Basile, Ragheb Moftah, Le Caire, 1998.

lui permettait de ralentir l'émission sonore. Elle a alors pris soin d'indiquer le tempo, les notes chantées plus bas ou plus haut que celles de l'échelle tempérée et tous les ornements - qui se distinguent visuellement de la mélodie par des caractères plus petits.

Cette transcription prend en compte le chant d'un seul chantre copte, Sadiq Attallah (1918-2001), élève de Mikhail Girgis, qui fut enregistré par Ragheb Moftah et dont l'exécution des mélodies a été intégralement notée avec tous les ornements réalisés. Sont également intégrés à l'ouvrage les textes de la liturgie en copte, en arabe et leur traduction en anglais, ainsi que le moment liturgique auquel les chants se rapportent. Cette transcription n'a pas pour vocation de permettre l'apprentissage des mélodies. Elle constitue en revanche un exemple des ornementations possibles, déjà réalisées par un chantre choisi pour sa fidélité envers les pratiques dites «traditionnelles » de la liturgie. C'est en ce sens que ce type de notation se prête au travail de sauvegarde : «Ainsi sera réalisé le rêve de R. Moftah : préserver ce corpus unique 
de musique traditionnelle $"{ }^{19}$ L'objectif n'était pas, en revanche, de fixer la mélodie en imposant son exécution avec tel ou tel type d'ornements. Elle fait simplement office de trace écrite de la pratique musicale copte au $X X^{\mathrm{e}}$ siècle.

Éditée en 1998 avec la contribution I'Université américaine du Caire où travaillait également $M$. Roy, cette transcription bénéficie à présent d'une diffusion au sein des milieux universitaires.

\section{INSTITUTIONNALISER ET DÉVELOPPER LA TRANSMISSION ORALE}

La transmission constitue un deuxième enjeu de poids dans cette démarche de sauvegarde de la musique copte. Elle marque en effet le lien entre une volonté de préserver et celle de faire connaître, de promouvoir et de perpétuer la pratique. Ce processus de transmission tend depuis quelques années à s'institutionnaliser à travers la création d'écoles et d'instituts, mais aussi par le biais du développement de diverses techniques d'apprentissage.

Au Caire, deux grands instituts proposent des cours : I'Institut d'Études Coptes et I'Institut Didymos. Leur vocation respective est différente et, partant, le public concerné n'est pas le même. À côté de l'Institut d'Études Coptes, des cours destinés à un public plus large se tiennent également au Collège Clérical. Enfin, un enseignement des chants est proposé dans les églises coptes du Caire, comme c'est le cas à Shubrâ. ${ }^{20}$

\section{Cours de I'Institut d'Études Coptes et du Collège clérical}

L'Institut d'Études Coptes est un établissement qui a vu le jour en 1954. La situation politique et sociale de la communauté copte à cette époque n'est pas étrangère à la constitution d'un tel établissement. Après 1918, les Coptes s'investirent dans le parti nationaliste égyptien et beaucoup intégrèrent le Wafd, parti indépendantiste. Leur action fut déterminante dans le processus qui conduisit en 1922 à l'indépendance formelle, puis en 1936-1937, officielle de l'Égypte. L'importance de cette communauté dans la vie politique égyptienne prit ainsi peu à peu forme. Toutefois, au début des années 30, des groupes de Musulmans, identifiant le nationalisme à I'Islam, voulurent faire de l'Égypte une nation islamique. Par la suite, sous le nouveau régime de Nasser, de 1953 à 1970, les Coptes n'eurent plus aucun impact sur la vie politique égyptienne. Certains choisirent alors d'émigrer en Europe ou aux États-Unis,

19. Martha Roy, 1992, "Liturgie Copte et enregistrements du Congrès du Caire ", Musique arabe - le Congrès du Caire de 1932, Le Caire, CEDEJ, p. 39.

20. Quartier nord du Caire, majoritairement chrétien. 
jetant ainsi les bases d'une diaspora qui n'en finit pas de s'accroître. ${ }^{21}$ Dans ce cadre, la création d'un organisme, I'Institut d'Études Coptes, spécialiste de la «question copte», leur a permis d'orienter positivement le sort de leur communauté, en lui offrant la présence d'une instance universitaire spécialiste de la culture copte. Cet aspect se poursuit encore de nos jours en dépit des discriminations dont les Coptes font encore parfois l'objet. Originellement, le but était de rassembler tous les écrits concernant la culture Copte, puis de les publier par le biais des «Cahiers Coptes». Cet Institut est maintenant un centre de formation, d'information et de documentation.

Dès son ouverture, une section d'études réservée à la musique y a été créée. Le premier responsable en a été Ragheb Moftah. Son successeur, Michael Ghattas, tient à poursuivre son œuvre. Des cours sont ainsi dispensés à des élèves dans le cadre d'une formation universitaire.

Parallèlement, M. Ghattas autorise le travail de plusieurs chœurs, indépendamment du cursus universitaire, au sein de l'établissement. Les chanteurs peuvent ainsi suivre les cours du mucallim Gad, chantre aveugle ${ }^{22}$, également professeur à I'Institut Didymos présenté plus bas. Quatre groupes viennent ainsi apprendre et parfaire leur exécution des chants. Ces actions s'inscrivent désormais dans une pratique sociale qui dépasse le cadre liturgique.

II en est de même pour les cours du Collège Clérical, fondé en 1894 sous l'impulsion du pape Cyrille V (1874-1927). Cette école se trouve dans I'enceinte de la cathédrale saint Marc du Caire, auprès duquel est I'Institut d'Études Coptes. La formation musicale proposée est dispensée par Ibrahîm 'Ayâd, chantre de renom chez les Coptes. Les cours sont mixtes et les élèves de tous âges. Les séances s'apparentent à de véritables réunions où les Coptes partagent, le temps du cours, un intérêt commun pour leur musique liturgique.

\section{Cours à l'Institut Didymos, dit « l'école des chantres aveugles»}

Cet institut, situé à Shubrâ, est ouvert aux garçons qui souhaitent devenir chantre. Ils peuvent ainsi profiter d'un enseignement dès leur plus jeune âge avec un apprentissage directement lié à la pratique du culte. Initialement, cet établissement, fondé en 1893 à Mahmashah au Caire, visait à instruire les jeunes aveugles. Mais de nos jours, il n'est plus réservé aux garçons atteints de cécité. L'Institut Didymos se présente comme un centre de formation, qui pro-

21. Christian Cannuyer, Les Coptes, Belgique, Brepols, 1996, collection « Fils d'Abraham »; L'Égypte Copte - Les Chrétiens du Nil, Paris, Gallimard/Institut du monde arabe, 2000.

22. Chez les Coptes, comme chez les Musulmans d'Égypte, le statut des aveugles a toujours été considéré comme une distinction divine et leur permettait ainsi une insertion sociale. 
pose à la fois des cours et une éducation liée à la compréhension des offices. Les jeunes garçons passent ainsi la journée à I'Institut. Ibrahim 'Ayâd et le $m u^{c}$ allim Gad y enseignent, en plus de leurs cours à l'Institut d'Études Coptes.

\section{Les écoles de musique liturgique}

Il m'a été possible d'observer d'autres cours au Caire, qui prennent la forme d'une véritable catéchèse basée sur la musique liturgique. Ces cours sont dispensés par des bénévoles pour tous les fidèles, enfants ou adultes, désireux d'approfondir leur connaissance de la liturgie et des chants. Les uns sont une fois encore prodigués à l'Institut d'Études Coptes, et les autres dans certains quartiers cairotes, notamment à Shubrâ, dans l'enceinte des églises.

\section{L'Institut d'Études Coptes}

Deux sortes de cours sont organisés au sein de cet Institut : ceux qui dépendent du cursus universitaire, déjà évoqués, et ceux qui sont assurés par des bénévoles, en-dehors de tout cursus. Michael Ghattas, responsable de l'enseignement musical de l'Institut, ne savait pas qu'au sein de l'établissement, des cours de chants étaient assurés par des bénévoles à l'intention de fidèles désireux d'approfondir leur connaissance de la musique liturgique copte. C'est en effet de façon tout à fait libre et bénévole que Magda, par exemple, Copte d'une cinquantaine d'années, se charge d'enseigner ce qu'elle connaît des chants coptes à qui le souhaite. Elle dispense ainsi des cours à deux groupes d'adultes, deux fois par semaine, qu'elle initie également à la langue copte. Cette dernière n'est en effet plus parlée depuis de nombreuses années ${ }^{23}$, mais elle continue à être utilisée dans la liturgie. Une transcription en Arabe permet généralement aux adultes de suivre.

Les groupes sont restreints : seules deux à trois personnes par séance suivent avec assiduité les cours. Malgré une ambiance tout à fait cordiale, le sérieux est de mise. La moindre erreur ou hésitation est reprise par Magda, consciencieuse dans son enseignement. Les adultes présents sont plutôt débutants dans leur exécution des chants. L'enseignement se porte principalement sur les longues psalmodies des offices du soir. Ces derniers sont les Absalmudiyyah, du nom du livre des tassabih (hymnes) de l'église. Il existe une Absalmudiyyah annuelle et une autre du mois de Kiahk (avant Noël). Ces célébrations sont intégralement chantées et présentent la louange des saints,

23. La langue Copte a été interdite dès le XI siècle, sous les Fatimides. Les Coptes devaient officiellement parler arabe, mais ils gardèrent leur langue pour les offices, Edris Abdel-Sayed, 1995, Les Coptes d'Égypte - Les premiers Chrétiens du Nil, Paris, Publisud. 
les Théotokiyya (louanges de la Vierge) et diverses autres récitations, ce qui implique des offices d'une grande longueur. Les chants, hymnes et psalmodies nécessitent ainsi un apprentissage de longue haleine.

Cet apprentissage est proposé à des Coptes qui n'ont pas eu la possibilité de suivre un quelconque enseignement étant plus jeunes ou qui veulent simplement approfondir leurs connaissances. De petites classes, indépendantes, se développent ainsi à l'initiative de certaines personnes. La portée de cette formation est uniquement liturgique et tend à permettre aux fidèles de participer aux offices du soir, majoritairement réalisés par les moines et plus rarement maîtrisés par la communauté religieuse. Ces offices, qui nécessitent un dialogue constant entre les Chrétiens et le récitant, ne peuvent en effet être célébrés qu'avec des pratiquants connaissant les mélodies. C'est pourquoi I'apprentissage de ces dernières se développe, afin de permettre aux Coptes de renouer avec leur tradition liturgique et ainsi, de la perpétuer.

\section{Les cours de Shubrâ}

Shubrâ est I'un des quartiers nord du Caire à majorité chrétienne. Les églises y sont plus nombreuses que dans les autres faubourgs. L'enseignement proposé est réalisé dans l'édifice religieux ou dans des salles annexes. Une grande effervescence habite ces lieux en permanence, soit à cause des cours, soit à cause des rassemblements de prières ou des offices.

Des cours sont dispensés aux adultes une à deux fois par semaine. L'apprentissage est une fois encore axé sur les chants des Absalmudiyyah, les offices du soir. Les élèves sont une quinzaine par cours. Comme celles des enfants, les classes d'adultes sont réparties par sexe. Contrairement aux autres cours de l'Institut précédemment évoqués, les professeurs des adultes à Shubrâ ont recours à un certain type de notation musicale sous la forme de traits. Au fur et à mesure qu'ils la chantent, ils écrivent la mélodie au tableau. D'après eux, cette technique s'avèrerait très utile pour mémoriser les chants. Ils représentent également la psalmodie à l'aide de gestes, alternant ainsi signes rythmiques et mélodiques.

Une des difficultés dans l'apprentissage des chants Coptes est le passage brusque d'un mode à un autre. On le constate lors des cours. Cela constitue une part importante du travail : il est rare que les élèves parviennent dès le premier essai à changer très naturellement de mode. Les enchaînements sont ainsi particulièrement travaillés.

Les cours dispensés aux enfants sont répartis par sexe et par classes d'âge. Les femmes donnent cours aux filles et les hommes, aux garçons. À Shubrâ, les plus jeunes suivent l'enseignement dans l'église et les autres, dans des salles annexes.

$C^{\prime}$ est seulement à partir de l'adolescence que les professeurs ont recours à une notation en bâtons au tableau. Pour les enfants, c'est la mémoire auditive, stimulée par la répétition, qui est ciblée. Ils peuvent aller apprendre les chants à l'église dès leur plus jeune âge. 
Dans les cours des plus jeunes, les élèves apprennent les grands hymnes coptes, tel que le Trisagion. Le cours présente une alternance entre la répétition de l'hymne en entier, la répétition de phrases nouvelles et courtes et le passage d'un enfant chantant l'hymne seul devant les autres. Lorsqu'ils ont trois à six ans, la concentration est réellement difficile : ils ne connaissent pas la langue copte et doivent pourtant mémoriser des mélodies liturgiques la plupart du temps dans cette langue. Dès l'adolescence, un suivi sur support écrit des textes est proposé, avec apprentissage de la langue, écriture musicale sous forme de traits au tableau et répétition des phrases mélodiques.

L'enseignement dispensé aux garçons est sensiblement le même que celui des filles. Il montre toutefois quelques divergences. En premier lieu, les garçons apprennent à jouer des cymbales en même temps que les chants. Seuls les hommes sont en effet habilités à s'accompagner de ces instruments pendant les offices. Leur apprentissage est ainsi très nettement orienté sur la pratique liturgique et un chœur de jeunes garçons, chargé de chanter la partie du chantre, est généralement formé pour représenter la situation de la messe. Pendant les cours, une séparation est ainsi organisée, entre un groupe de garçons, qui exécute la partie du soliste et le reste des élèves, c'est-à-dire les fidèles. Ensuite, le niveau de formation des garçons est plus exigeant que celui des filles. Très tôt, dès sept à huit ans, ils apprennent les chants des Absalmudiyyah et de la Tasbeha. Ces chants de louanges présentent en effet de nombreuses difficultés, notamment par la présence de longues vocalises. La destination liturgique de cet apprentissage implique, de la part des jeunes garçons, une rapide maîtrise des textes et de leur psalmodie. La notation en bâtons est déjà utilisée par les enseignants dans ces cours.

D'une manière générale, et suite à mes observations, il apparaît que plus l'âge augmente, plus le nombre de procédés pour mémoriser les chants est important. Les plus jeunes apprennent les hymnes les plus connus et les plus exécutés qui sont, par conséquent, les plus faciles à mémoriser. Mais, mis à part ce cas particulier, il s'avère que la mémoire n'est plus utilisée seule : les Coptes utilisent ce qui, pour eux, sont des mécanismes récents de mémorisation des chants, tels que la transcription musicale. Comme nous l'avons déjà remarqué, rien ne prouve qu'il n'ait pas existé, par le passé, une forme quelconque de notation. Toutefois, à l'heure actuelle, l'éducation musicale des Coptes entraîne des processus d'apprentissage particuliers, par le biais de l'écriture et de la représentation visuelle. La mémorisation auditive demeure une large part de l'enseignement, mais elle s'exerce en parallèle d'autres mécanismes de transmission qui participent de cette démarche de patrimonialisation.

Ces modalités d'enseignement visent à parfaire et à assurer la viabilité de la connaissance de la musique copte. Dans la logique d'une musique accessible pour tous, les Coptes mettent ainsi en œuvre des techniques visant la transmission et la pratique des chants. Cet enseignement, développé dès le plus jeune âge, est l'un des vecteurs de la propagation de la musique liturgique. 


\section{L'ENREGISTREMENT : SES MÉTHODES ET LEURS OBJECTIFS}

L'enregistrement des musiques coptes est une entreprise qui est née sous I'impulsion de Ragheb Moftah. Elle s'est ensuite considérablement développée au fil du XXe siècle, grâce aux nouvelles technologies qui se sont démocratisées.

L'enregistrement a plusieurs objectifs : celui de conserver les chants coptes, celui d'en faciliter la transcription et celui de les diffuser.

\section{Le rôle pionnier de Ragheb Moftah : ses moyens et ses méthodes $\mathrm{d}^{\prime}$ archivage}

Définitivement reconnu par la communauté Copte comme le protecteur du patrimoine musical, le travail de R. Moftah apparaît comme le plus sérieux dans ce domaine, ainsi que l'exprime G. Kyrillos : " The huge and great effort in recording the Coptic hymns from the mouths of the noted and accurate early cantors on cassette tapes was a magnificent and splendid work. History will attribute that to Dr. Ragheb for his work will be the only genuine and trustee reference for he had fetched to us the raw melodies from their origin before doing any scientific reparation $»{ }^{24}$ L'objectif de R. Moftah était de rendre pérennes "les formes authentiques de la musique $" .^{25}$ L'idée était que les enregistrements soient réalisés à partir des détenteurs de la tradition musicale, transmise à travers les siècles. Ce type d'affirmations soutient le caractère exagérément ancestral et " pur » de la musique copte. Lorsqu'ils émanent de quelqu'un de respecté, de tels propos deviennent indiscutables pour les gens de la communauté. L'influence des universitaires crée ainsi la tradition musicale Copte : elle en devient dès lors « authentique ».

Lors du Congrès de musique arabe du Caire de 1932, R. Moftah a été invité à présenter la musique copte. C'est à cette époque que la décision d'enregistrer certaines parties de la Sainte Messe a été prise. D'autres bandes ont été réalisées au département de musique de l'Institut d'Études Coptes dès 1954. La démarche de R. Moftah avait été, entre temps, de réunir un bon matériel d'enregistrement, qui a pu être installé dans une pièce aménagée en studio au sein de l'Institut. C'est ainsi qu'il a disposé de son propre centre d'archivage, afin d'enregistrer les chants liturgiques. Il a ensuite demandé à des chantres d'interpréter les chants des différents offices dans leur intégralité. Le plus enregistré est sans conteste Mikhaïl Girgis el-Batanony. Les étagères sont emplies des bandes des enregistrements réalisés par R. Moftah dans la seconde moitié

24. George Kyrillos, Coptic hymnody, its Spirituality and Tunes, http://www.davidensemble.com/english/book.htm.

25. «[...] and making recordings of authentic forms of the music », in introduction de A transcription of the Complete Liturgy of St Basile, Ragheb Moftah, avec la collaboration de Margit Tóth et Martha Roy, Le Caire, 1998. 
du $X^{\mathrm{e}}$ siècle. La plupart sont disponibles sur cassettes. Le studio n'est plus réellement utilisé, sinon pour organiser des répétitions de chœurs. Par manque de moyens financiers, le matériel tombe en désuétude et n'est plus exploité.

\section{Les nouveaux moyens de diffusion de la musique}

La multitude d'enregistrements effectués à l'heure actuelle par des chœurs Coptes a contribué à développer un véritable marché de diffusion de la musique liturgique et extra-liturgique au sein de la communauté. Les productions discographiques sont réalisées par des groupes paroissiaux, qui se consacrent à la divulgation de la musique copte, généralement au sein même des églises. Face à ce nouveau marché, les stratégies de vente se développent, là aussi dans un cadre qui reste religieux.

Il est ainsi possible de trouver, dans l'enceinte de chaque église, des points de vente d'objets en tous genres, relatifs à la religion. Parmi eux, on trouve des compact-discs, des cassettes, des DVD, des cassettes VHS et des cédéroms. Leur contenu concerne la musique liturgique, mais pas seulement. On peut, en effet, constater la présence d'enregistrements de compositions modernes de musique, de cours donnés par des chantres, de concerts organisés lors de manifestations religieuses, de films, etc. Un aspect ludique existe aussi pour les enfants, avec un développement de disques, de films et de dessins animés liés au catéchuménat. La vente d’objets multimédias trouve ainsi son essor dans la diffusion auprès des fidèles, particulièrement friands de ces nouveaux supports.

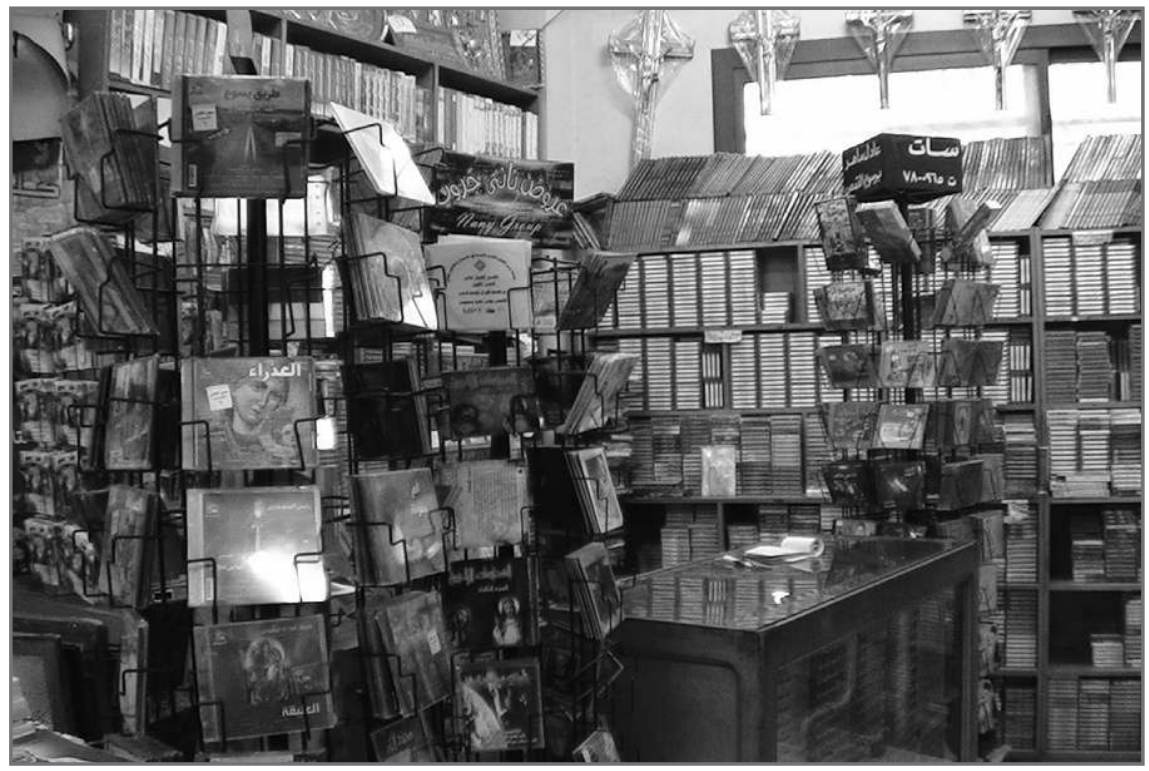

3. Étalage de disques, cassettes audio, vidéos et DVD dans la boutique du Moqatam (lieu de pèlerinage, Le Caire, février 2007, Severine Gabry). 
De nombreux sites Internet se chargent également de diffuser la musique copte. Pour la plupart, ils sont développés par les diasporas et s'attachent à présenter les pratiques musicales, toujours sous les auspices d'une authenticité revendiquée. ${ }^{26}$

La sauvegarde de la musique copte est ainsi devenue l'une des priorités de cette communauté depuis la fin du XIX ${ }^{\mathrm{e}}$ siècle. C'est à travers différents types de transcriptions, la transmission et les nombreux enregistrements disponibles que les Coptes s'engagent à préserver leur musique, afin de la protéger de sa dégradation présumée.

\section{ENJEUX DE LA PATRIMONIALISATION DE LA MUSIQUE COPTE}

Tous les enregistrements, transcriptions, colloques et manifestations diverses entourant le patrimoine musical copte sont à présent répertoriés et peuvent faire l'objet d'une historiographie. Ils sont ainsi exposés dans des ouvrages qui, à l'aide de dates et de noms précis, permettent de constituer une histoire de la patrimonialisation de la musique copte âgée de plus de cent ans. Mais pourquoi et comment la musique est-elle devenue un élément de revendication identitaire ? Elle a en effet été le terrain privilégié de toutes sortes de processus de patrimonialisation et il convient de comprendre en quoi elle a incarné, à travers eux, un élément incontournable de la définition de la tradition copte.

La musique permet l'utilisation de moyens qui contribuent au discours identitaire des Coptes en mettant en lumière une stabilité des pratiques. Justifier que la musique est ancestrale assure en effet une pérennité et une fixité au langage musical copte et, par conséquent, à la liturgie.

Comme j'ai pu le constater, et comme il en a été question ci-dessus, ces moyens se traduisent par l'abondance de transcriptions, d'enregistrements et par la création d'institutions et d'écoles. Ces dernières, par l'enseignement, permettent une diffusion de la musique auprès d'un large public, procédé qui se développe depuis maintenant un demi-siècle. La transcription est précisément le processus qui a soulevé le plus d'interprétations. G. Villoteau, J. Blin, L. Badet et R. Moftah sont quatre personnes à avoir envisagé la musique copte comme I'héritière des musiques pharaoniques et à avoir pris cela comme argument pour mettre en place une démarche de sauvegarde des chants liturgiques. La stabilité présumée de la pratique musicale en tant que pratique cultuelle - l'Église copte se dit en effet très conservatrice - est l'argument qui permit à ces quatre transcripteurs d'asseoir leur théorie à propos des origines de la musique copte.

26. Cf. bibliographie. 
G. Villoteau, dans sa contribution à la Description de l'Égypte ${ }^{27}$, cherche à donner une vision globale des musiques pratiquées en Égypte et parmi elles, de la musique copte. Son but est donc de faire connaître, par le biais de la musique, une facette de la culture chrétienne égyptienne. Il qualifie toutefois cette musique de "fastidieuse ", « ennuyeuse ", «monotone», " insignifiante » et autres qualificatifs aussi peu élogieux les uns que les autres. G. Villoteau estime en effet que "s'il s'était conservé en Égypte quelques restes de l'ancienne musique de ce pays, de laquelle Platon nous a tant vanté la merveilleuse perfection, nous eussions dû les retrouver dans les chants des Qobtes $» .^{28}$ Ce ton laisse présumer sa déception lors de la découverte des chants coptes, réalisés par "des gens stupides » qui n'ont pas su, selon lui, conserver leur belle musique antique. II n'a en réalité pas pris la peine de transcrire sérieusement ne serait-ce qu'un chant copte. Avouant lui-même s'être endormi pendant les écoutes répétées de la mélodie, que penser de cette transcription ? Toutefois, son objectif était bien, initialement, de faire connaître la musique copte et de la mettre en valeur par rapport à son passé prestigieux. Il s'agissait ainsi de souligner le caractère récurrent et stable de la pratique musicale liturgique afin de justifier son aspect traditionnel. Ce travail n'est pas reconnu par les Coptes qui gardent en mémoire le jugement peu gratifiant de G. Villoteau. Si eux-mêmes cherchent à valoriser leur patrimoine musical, ce n'est certes pas avec de tels avis qu'ils peuvent y prétendre.

Les essais suivants sont du Père J. Blin en 1888. II valorise beaucoup son travail : " Le travail que nous offrons au public ne sera pas d'une médiocre utilité pour la nation copte ». II y précise que les Coptes n'ont jamais noté leurs chants, que seule "la tradition a conservé leur musique $»{ }^{29} \mathrm{Il} \mathrm{critique} \mathrm{impli-}$ citement G. Villoteau en réfutant les termes que ce dernier avait employés à propos de la musique copte (" mélodies sauvages et soporatives ») et précise $\mathrm{qu}^{\prime}$ « ils n'ont rien de la rudesse de la mélodie arabe. Leurs cadences et leurs rythmes, plutôt gais que tristes, ne respirent pourtant point une gaieté qui naît du plaisir. On trouvera, surtout à partir de la préface de la messe, $n^{\circ} 31$ jusqu'à la fin de l'office, des passages qui rappellent les belles modulations de notre plain-chant ». ${ }^{30}$ Jules Blin véhicule ici l'idée d'une musique unique qui n'a rien à envier au plain-chant occidental et qui ne ressemble en rien aux mélodies arabes. Il est également joint, dans son ouvrage, une lettre de Son Eminence le Cardinal Simeoni, préfet de la Sainte Congrégation de la Propagande, datée du 8 février 1888 : «Votre Révérence aura ainsi bien méritée de l'Église copte ;

27. Guillaume André Villoteau, 1826, Description de l'Égypte, Paris, t. XIV.

28. Ibid., p. 754.

29. Jules Blin, Chants liturgiques Coptes, Partie chantée par le peuple et le diacre, Le Caire, Imprimerie nationale, 1888.

30. Ibid., préface. 
elle lui aura conservé le chant liturgique qui allait en se corrompant et menaçait de se perdre. Son travail, en outre, permettra au Révérend Père Foujols et à ses successeurs de faire enseigner, à tous les élèves du séminaire Copte, le chant en même temps que les cérémonies du rite, conformément à ce qui a été ordonné par cette Sainte Congrégation, en Avril 1878 ».31 J. Blin appuie luimême ce discours en affirmant que les chants religieux conservent en général «l'empreinte nationale», sous-entendu, l'empreinte de l'Égypte ancienne. Selon lui, « ils ne semblent pas avoir, contrairement aux chants profanes, une physionomie et des allures étrangères $»{ }^{32}$ La musique apparaît ici comme exempte de toute influence et " purement » copte. Cette idée est en parfaite concordance avec l'idéal identitaire des Chrétiens d'Égypte. Toutefois, cet ouvrage reste peu connu au sein de la communauté. J. Blin ne figure pas parmi les noms cités par les Coptes pour désigner les précurseurs de l'archivage musical liturgique. Cette initiative catholique, à dessein vraisemblablement prosélyte, n'a probablement pas été reçue favorablement par la communauté copte orthodoxe de l'époque. La démarche n'émanant pas d'elle-même, mais de missionnaires occidentaux, le travail réalisé n’a pas été pris en compte.

Le Père L. Badet, de la Compagnie de Jésus, a lui aussi réalisé un travail de transcription de chants Coptes en 1899. Les mélodies transcrites sont accompagnées d'un descriptif du culte ainsi que des textes énoncés. C'est une fois encore dans la préface que se dessine tout I'intérêt, pour L. Badet, de noter ces chants. Comme son prédécesseur J. Blin, il considère que seule la notation pourra pallier les problèmes de dégradation de la musique liturgique Copte : "La notation des chants liturgiques coptes est nécessaire, si I'on veut les conserver dans leur intégrité $»{ }^{33}$ II remarque que les chants ne sont pas chantés partout de la même manière et l'explique en disant qu'avec la transmission de bouche à oreille, ils n'ont pas pu être retenus fidèlement. II suppose toutefois que les mélodies ont gardé « leur cachet spécial ». L'avenir qui attend la musique copte dépend aussi, selon L. Badet, de la musique européenne, qui « a fait invasion à son tour, accentuant par l'harmonie le mélange du chromatisme et du diatonisme, et faisant ainsi de l'altération et de la cadence parfaite comme un besoin de l'oreille $»{ }^{34}$ Ce point de vue implique la reconnaissance $d^{\prime}$ influences dues à la proximité de cette musique avec d'autres. Ce discours sert l'idée du caractère ancestral du chant copte. Préservés jusqu'à présent, ils seraient menacés d'être pervertis par la musique occidentale. C'est pour

31. Ibid.

32. Ibid.

33. Louis Badet, Chants liturgiques des Coptes, notés et mis en ordre par le Père Louis Badet de la Cie de Jésus, Le Caire, Collège de la Sainte Famille, Petit séminaire Copte, 1899.

34. Ibid. 
pallier ce problème que L. Badet se charge de cette tâche de transcripteur. Son but est aussi de permettre aux prêtres d'apprendre les chants à partir de cette trace écrite : "On le voit, la transcription des chants liturgiques coptes, non seulement arrêtera leur décadence, mais même aidera à les exécuter avec plus de perfection $» .{ }^{35} \mathrm{Il}$ ajoute qu'il est absolument nécessaire de donner des notions musicales aux séminaristes et inclut ainsi dans son ouvrage une partie consacrée aux "Principes élémentaires de la musique». Un large éventail de techniques est mis en place pour aller à l'encontre de la perte de la musique copte. Bien qu'élogieux envers l'Église chrétienne d'Égypte, son art et ses pratiques, les Coptes ne reconnaissent pas non plus ce travail, puisqu'il renvoie aux mêmes objectifs missionnaires que J. Blin.

Ces trois exemples du XIXe siècle partaient d'une réelle volonté de sauvegarder la musique copte, tout en la faisant passer pour un objet resté invariable des années durant. Le prétexte était de conserver les restes décadents d'une très ancienne musique (toutefois déjà perdus selon G. Villoteau) et de répandre par ce moyen la culture copte. La stabilité de la pratique musicale en tant que pratique cultuelle est l'argument qui permit à ces trois transcripteurs d'envisager, de prime abord, la musique copte, même si on constate une différence importante de jugement entre G. Villoteau et les deux missionnaires.

Ce sont les travaux de R. Moftah qui sont de loin ceux auxquels la communauté copte attache la plus grande importance. Au regard des chrétiens d'Égypte rencontrés en février 2007 au Caire, seuls les Coptes eux-mêmes ont pu réaliser une étude crédible de leur musique. Que R. Moftah soit Égyptien a en outre une importance cruciale. Sa démarche repose, comme celle de ses prédécesseurs non égyptiens, sur la volonté de préserver et de faire connaître ce qu'est la musique liturgique copte. Au-delà de toutes considérations musicales à proprement parler, $c^{\prime}$ est la culture copte qui est mise en avant dans ses travaux. Grâce aux moyens qu'il a mis en œuvre, certains aspects musicaux participent à la définition de la réalité liturgique copte. La préface de l'ouvrage déjà cité $A$ transcription of the Complete Liturgy of St Basile présente à ce titre un grand intérêt. La musique de l'Église copte orthodoxe est une fois de plus valorisée en tant qu' « unique dans la musique liturgique du Moyen Orient ». ${ }^{36}$ Elle est donc dite à part des traditions arabes, arméniennes, hébraïques et grecques de la région. D'après ses dires, la communauté copte aurait conservé ses traditions au fil des années : certains chrétiens égyptiens pensent que c'est à travers la performance musicale et la mémoire que cette démarche fut possible. Le livre dirigé par R. Moftah a le dessein suivant : vouer la musique et les

\section{Ibid.}

36. "The music of the Coptic Orthodox Church is unique in the liturgical music of the Middle East », in introduction de A transcription of the Complete Liturgy of St Basile. 
textes des Coptes à la postérité, ce qui est un point important « dans I'histoire $\mathrm{d}^{\prime}$ une grande et noble Église, à la gloire de Dieu $»{ }^{37}$ Le processus de conservation a ainsi pris une grande ampleur avec R. Moftah : la musique, par son originalité, représente et préserve la culture copte.

Ces divers exemples montrent que la musique copte est considérée actuellement comme ayant été depuis au moins deux mille ans un vecteur de la pratique liturgique. À présent, la tradition se trouve perpétuée par les transcriptions, qui témoignent de la stabilité présumée dont aurait fait preuve la musique copte pendant plus de deux millénaires.

\section{CONCLUSION}

La musique est le terrain privilégié des processus de patrimonialisation de la culture copte parce qu'elle marque un lien tangible, aux yeux du monde, avec son glorieux passé. Celui-ci se décline principalement sous le jour de I'héritage de la musique pharaonique. Aux yeux de la communauté copte, cette " origine » de la musique liturgique justifie son discours et lui permet de penser que la musique est une tradition qui, effectivement, a un passé ancestral, voué à la pérennité grâce au culte et à la foi. E. Hobsbawm dit à propos des traditions qu' « elles tentent normalement d'établir une continuité avec un passé historique approprié ${ }^{38}{ }^{38}$ La musique liturgique Copte, par son lien avec l'histoire de l'Égypte, entre ainsi dans la catégorie de ces arts qui se prêtent au discours identitaire du peuple qui les exécute, en les légitimant à I'aide d'un passé mythique. II n'est pas dans l'ordre des choses de changer ce que les Coptes ont reçu de leurs pères. La musique copte, dite " traditionnelle », est ainsi devenue le symbole de sa communauté par diverses actions engagées tout au long du XXe siècle : la récurrence des pratiques, par le biais de l'enseignement pour tous et des offices religieux ; la stabilité, par l'intermédiaire d'une notation qui assure une pérennité et une fixité au langage musical Copte ; I'authenticité rendue accessible, par l'action de groupes et de chorales qui adhèrent à la réalisation d'une musique "pure », et qui s'attellent à une production de disques destinés à tous.

Les processus de patrimonialisation se déploient ainsi au sein de la communauté copte depuis près de 150 ans. La transcription, premier processus mis en place, endosse divers rôles : sauvegarder, proposer des traces présumées de la musique pharaonique et analyser le matériau musical copte actuel. La notation des chants coptes, par ses divers objectifs, suscite ainsi un large intérêt

37. " [...] in the history of a great and noble Church, to the glory of God », Id.

38. Eric Hobsbawm, Terence Ranger, 2006, L'invention de la tradition, Paris, éd., Amsterdam, p. 12. 
par la multitude d'interprétations qu'elle offre. Coptes et non Coptes se sont investis dans ce travail, mais seuls les travaux lancés par des chrétiens d'Égypte ont un impact auprès de la communauté. Il est en effet intéressant de constater que les transcriptions des missionnaires catholiques J. Blin et L. Badet, par exemple, ne sont pas ou très peu évoquées par les Coptes. À cet égard, seuls les travaux dirigés par $\mathrm{R}$. Moftah, connus de tous, ont un réel impact dans la conscience musicale de la communauté.

Dans le domaine de la transmission, il m'a été possible de relever la mise en œuvre de différents processus : I'apprentissage, grâce aux instituts et aux écoles, les enregistrements et la démocratisation des moyens de diffusion de la musique. L'éducation musicale en école ou en institut propose différentes techniques d'enseignement pour tous les âges. Les enregistrements et le développement de moyens de diffusion permettent aux Coptes d'avoir facilement accès aux musiques liturgiques, ce qui concourt à développer la transmission et la réception de ces musiques, par imprégnation. Il est intéressant de constater que ces processus participent à la propagation de la musique copte, mais aussi qu'ils explorent de nouveaux horizons musicaux, autres que liturgiques, au sein de la communauté. Deux conséquences se dégagent ainsi de ces mécanismes de transmission: la patrimonialisation d'une musique dite « traditionnelle » par un apprentissage visant à la pérenniser et l'élaboration d'une nouvelle musique qui se dégage du cadre liturgique. Dans les deux cas, ces mécanismes ont pour objectif de diffuser des chants et des hymnes coptes. À travers cet acte conscient de transmission/réception, nombre de chrétiens d'Égypte s'appliquent ainsi à déterminer le sens de leur tradition.

Ces deux processus majeurs s'inscrivent dans une actualité qui vise à définir une tradition musicale copte. Celle-ci englobe ainsi différents aspects : une tendance à l'autarcie culturelle, une conscience historique très forte, un sens de la continuité « ethnique », une tradition de la manière même de penser et aussi une conception dynamique de la religion. Dans cette communauté, les liens entre tradition musicale et tradition religieuse sont en effet étroits.

À cet égard et par le biais de sa pratique, la musique copte offre un large terrain d'investigations sur la mise en valeur de la tradition. Son passé en Égypte lui confère une signification que les Coptes utilisent à des fins identitaires, propice aux revendications. "La tradition définit un topos à la fois imaginaire, symbolique et géographiquement bien délimité, balisé par un nomos ou une règle' $»{ }^{39}$ Les Chrétiens d'Égypte cherchent à restaurer un ordre ancien pour légitimer l'authenticité de leur musique et pour défendre ainsi leur identité. C'est le matériau musical qui est revendiqué comme traditionnel : sa transmission et sa transcription mettent en valeur son caractère ancestral et se sont

39. Jean During, 1994, Quelque chose se passe - le sens de la tradition dans l'Orient musical, Lagrasse, Verdier, p. 16. 
ainsi considérablement développées. Par leurs actions, elles ont fait ellesmêmes évoluer la notion de tradition au sein de la musique liturgique copte, qui exalte la religion et l'autochtonie de sa communauté. À ce sujet, il apparaît une volonté certaine de se démarquer d'autres cultures, principalement de celle des Musulmans.

Les Chrétiens d'Égypte ont ainsi décidé du poids et du sens à donner à leur musique traditionnelle liturgique par différents processus de patrimonialisation. Quant aux nouvelles musiques créées, à l'écart des manifestations liturgiques, elles marquent une évolution de la notion même de " tradition » chez les Coptes. Par les créations musicales d'un côté et les revendications nationalistes de l'autre, il s'agit là d'un compromis entre modernité et tradition choisi plus ou moins consciemment par les Chrétiens d'Égypte.

\section{RÉFÉRENCES BIBLIOGRAPHIQUES}

Abdel-Sayed E., 1995, Les Coptes d'Égypte - Les premiers Chrétiens du Nil, Paris, Publisud.

Atıya A. S., 1991, the Coptic Encyclopedia, New York, Maxwell Macmillan International, 8 volumes.

BADET L., 1899, Chants liturgiques des Coptes, notés et mis en ordre par le Père Louis Badet de la $C^{\text {ie }}$ de Jésus, Le Caire, Collège de la Sainte Famille, Petit séminaire copte.

BuIN J., 1888, Chants liturgiques des Coptes, Le Caire, Imprimerie Nationale, $95 \mathrm{p}$.

Borsal I., 1968, "Mélodies traditionnelles des Égyptiens et leur importance dans la recherche de I'ancienne musique pharaonique », Acta Antiqua Academiae Scientarium Hungaricae 10, p. 69-90.

Cannuyer C., 1996, Les Coptes, Belgique, Brepols, collection « Fils d'Abraham », 2.

DurING J., 1994, Quelque chose se passe - le sens de la tradition dans l'Orient musical, Lagrasse, Verdier.

Hickmann H., 1980, Vies et travaux I, Le Caire, Organisation des Antiquités de l'Égypte, service des musées, Miscellanea Musicologica.

Hobsbawm E., Ranger T., 2006, L'invention de la tradition, Paris, Ed. Amsterdam. Marsot A. L. S. , 1984, Egypt in the reign of Muhammad Ali, Cambridge, Cambridge University Press.

MÉnARD R., 1952, "La musique copte, problème insoluble? », Les Cahiers Coptes, $n^{\circ} 1$, p. 35-40.

MoftaH, R. H., 1998, A transcription of the Complete Liturgy of St Basile, Le Caire, avec la collaboration de Margît Tóth et Martha Roy, Presse de I'Université Américaine. 
Moussalı B., Les enregistrements du Congrès de Musique arabe du Caire, recueilli et édité par Jean Lambert. À paraître.

Vigreux P., 1992, Les documents du premier Congrès sur la musique arabe, Le Caire, 1932, Le Caire, CEDEJ.

Villoteau G. A., 1826, Description de l'Égypte, Paris, Impr. Impériale, tome XIV, chapitre V.

Volle B., 2004, Les Coptes d'Égypte sous Nasser - Sainteté, miracles, apparitions, Paris, CNRS Éditions, 288 p.

Sites internet :

George Kyrillos, Coptic hymnody, its Spirituality and Tunes, à consulter sur le site :

http://www.davidensemble.com/english/book.htm

http://eocf.free.fr/sites.htm

http://www.davidensemble.com

http://www.coptic.org/

http://www.coptichymns.net/

http://tasbeha.org/

http://www.suscopts.org/deacons/hymns/hymns.html

http://www.egypteeternelle.net/papyrus/v01 n06/article1.htm

http://www.geocities.com/Athens/Delphi/7261/copticmusic.htm

http://www.coptic.org/music/nota.htm 